26

\title{
CONSENT IN CLINICAL RESEARCH
}

\author{
Collin O'Neil
}

\subsection{Introduction}

The defining goal of clinical research is to generate knowledge that can be used to enhance the therapeutic capacities and decision-making of clinicians and policymakers. Achieving this goal often requires experimenting on or collecting information about human subjects ${ }^{1}$ in ways that would, without consent, infringe their rights. Guidelines for human subjects research, such as the Nuremberg Code, the Declaration of Helsinki, the Belmont Report, and the Common Rule Regulations exist because of notorious studies that, among other things, failed to obtain voluntary and informed consent from human subjects. The Nazi doctors forced prisoners into harmful experiments (Claims Conference 2007). The Tuskegee Syphilis Study tricked subjects into accepting a painful non-therapeutic lumbar puncture under the guise of "special free treatment" (Brandt 1978). Had the investigators in these experiments sought voluntary and informed consent, subjects would have refused and avoided serious harm. But protection from unwanted harm is not the only function of consent. In another much-discussed study, patients at the Jewish Chronic Disease Hospital were injected with live cancer cells for a purely scientific purpose, without being told that the injection was not for their benefit and that it contained live cancer cells (Arras 2008). The researchers argued that, since the injection posed no serious risk, the patients did not need to be informed about the contents or purpose of the injection to protect themselves from harm. Nevertheless, by injecting the patients without their informed consent, the researchers had clearly infringed on the patients' rights to decide what is done to their bodies.

The lessons of these early scandals have been learned. The norm of obtaining voluntary and informed consent is now deeply entrenched in clinical research. Yet the topic of consent in clinical research remains a focus of discussion. There are two principal areas of continuing controversy. One centers on the question of when consent to low-risk research is necessary and when it is not. It can be very costly or even infeasible to seek consent for certain types of studies, such as research on biological samples and emergency research. If consent is unnecessary in such studies this would be a boon to science, but there is disagreement about the conditions under which low-risk research may go forward without consent.

Another source of controversy derives from a worry that, even when consent is sought from subjects, the consent they provide is often defective. Consent should be adequately informed and voluntary. But empirical research has shown that subjects frequently have deficits in their 
Collin O’Neil

understanding of the studies they are participating in, especially those aspects that set research apart from the patient-centered settings they are accustomed to (Mandava 2012). Furthermore, researchers routinely offer money to healthy individuals and sometimes even to patients as an incentive to enroll in their studies. Many ethicists and institutional review board (IRB) members are concerned that these payments and other incentives such as free health care may be compromising the voluntariness of the consent that researchers obtain.

This article begins with an overview of several types of clinical research that have sparked debates about the necessity of consent, followed by a brief discussion of the considerations relevant to resolving those debates. The focus will then turn to the question of what conditions must be satisfied for consent to be effective. This is the issue of the "validity conditions" for consent to research. The earliest and still most influential account of these conditions is non-moralized, in the sense that the validity conditions make no essential reference to whether the researcher soliciting consent from a subject has obtained it in a way that wrongs the subject or not. Recently new approaches that moralize some of these conditions have been put forward. As we'll see, the non-moralized and moralized approaches to consent in research yield very different implications for how we should evaluate the use of payment and other incentives to increase enrollment and how troubled we should be by the data indicating that subjects often misunderstand various aspects of study design.

\subsection{When is consent to research necessary?}

Consent communicates an intention to make it the case that someone else would not wrong you by treating you in a certain way (Owens 2012: 165). That is, it purports to waive a right. When consent is "valid", it successfully waives that right. When a researcher proposes to treat a subject in a way that would infringe a right without consent, then obviously consent is necessary to avoid infringing that right. But if the researcher plans to involve the subject only in ways that would not wrong the subject without their consent, there are no rights that need to be waived. Since seeking consent can impede the progress of research, it is important to get clear on exactly what ways of involving a subject in research would infringe on their rights without their consent.

There are some types of clinical research that are routinely conducted without obtaining consent. Epidemiological research on medical records, for example, dispenses with consent. So long as confidentiality is protected, this practice is fairly uncontroversial (Miller 2010). This may be in part because this research does not include interventions on human subjects. But storing patients' tissues or blood for research purposes (after they have been removed, with consent, for therapeutic reasons) does not intervene on the bodies of subjects themselves either, nor does any of the research that is subsequently conducted on those tissues. Yet many believe that storing those samples for research purposes requires consent, and some even argue that subjects must be re-consented for every study that is subsequently done on those samples, even though this would discourage valuable research (for discussion, see Grady 2015).

There is also clinical research that does intervene on the bodies of human subjects and yet proceeds without consent from the subject or even a surrogate. Emergency research randomizes subjects with acute and life-threatening illnesses between experimental and standard emergency care. This is sometimes done without their consent (and without the consent of a proxy) not only when consent would be impossible to obtain in time, but also when prospective consent would be possible but would greatly reduce the enrollment rate or lead to imbalanced enrollment (Karlawish 2008). Randomized controlled trials comparing two approved and widely used treatments typically do seek consent to research from the subjects, but recently some 


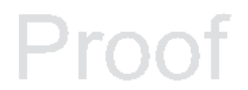

Consent in clinical research

ethicists have argued that consent to the particular treatment they receive suffices and that consent to research is unnecessary (Faden et al. 2014).

At least three considerations are relevant to settling the question of whether consent to research must be obtained in a given study. The first is whether there are any rights that the research would infringe without consent. Do subjects have any rights against the use of their biological samples for research purposes, assuming there are already safeguards in place to protect them from embarrassing or harmful disclosures? If they do have privacy or perhaps property rights in relation to those samples, then the mere fact that the risks are very low would not be enough on its own to justify dispensing with consent. But if they do not, or if there are ways to work around those rights (e.g., by anonymizing the samples), there would be no need for consent. Regarding comparative effectiveness trials, do subjects have a right not to be experimented upon that is distinct from their right not to be treated without consent? If they do then consent to research may be necessary (O'Neil 2014), but if not then consent to the particular treatment they receive would suffice.

When the research would infringe a right without consent, the second factor to consider is the strength of the right and the opportunity costs of seeking consent. Rights may sometimes be justifiably infringed. If the pertinent rights are weak, the fact that obtaining consent would slow important scientific progress could be enough to override them (Miller 2010). But whether this fact is indeed enough to override them may also depend on a third factor, namely, whether the justification treats subjects' hypothetical consent as a constraint. Some justifications for dispensing with consent procedures do not presuppose that anyone would refuse if asked. The reason for dispensing with consent is only that the process of seeking consent would require so much time and resources that the research would no longer be worthwhile. ${ }^{2}$ But other justifications do presuppose that there would be high or imbalanced rates of refusal. Both kinds of justifications cite impediments to scientific progress, but they differ in their relation to hypothetical consent. The former justification is consistent with recognizing hypothetical consent as a constraint on human subjects research. The legitimacy of the latter justification is highly questionable because it flouts that constraint. Not only does it anticipate that subjects would not consent if asked, that is the very basis of the justification.

\subsection{When is consent to research valid?}

Once we are convinced that a study involves a subject in a way that would, without the subject's or a surrogate's consent, infringe a right, a new question arises. Under what conditions is consent effective in making it the case that the subject is not wronged by that involvement? Here it is important to distinguish between failures that are due to limits on the power of consent and failures that are due to defective consent. It may be the case that consent, no matter its quality, is incapable of cancelling duties to subjects not to expose them to serious risks of harm for a trivial reason (Tadros 2016: 265-80), to transact with them on terms that distribute the benefits fairly (Wertheimer 2011: 191-254), and to provide them with certain forms of ancillary care (Richardson 2012: 133-43). ${ }^{3}$ In what follows I set aside issues about the limits on the power of consent and ask only, when a right is waivable by consent, under what conditions is consent effective in waiving the right? This is the question of the "validity conditions" for consent to research.

\subsection{Competent consent}

One condition that must be met for an act of consent, on its own, to waive a right against an intervention is that the consenter be competent with respect to the decision: that is, possess 
sufficient decision-making capacity to make the decision. Decision-making capacity is the capacity to make a decision that best reflects one's values. This capacity calls on both cognitive abilities to form a judgment about what one ought to do in light of the facts, and volitional abilities to form, maintain, and execute an intention that reflects that judgment. Young children are regarded as incompetent to waive their rights against involvement in research. Adults who suffer from advanced dementia may have cognitive impairments that render them incompetent to consent to research, and adults suffering from severe depression or addiction may have volitional impairments that render them incompetent to consent to certain kinds of research. Findings of incompetence are relative to the decision being made. Arguably an adult who has sufficient capacity to make a decision about treatment may not have sufficient capacity to make a similarly complex decision about research, if they are incapable of appreciating the relevant differences between clinical care and research (Rosenstein \& Miller 2008).

What is the upshot of a determination of incompetence? Although the Nuremberg Code appears to rule out experimenting on anyone who is incompetent to consent to research, current guidelines and regulations permit some experimentation on incompetent subjects, so long as consent is obtained from a surrogate. Also, although incompetent subjects cannot by themselves effectively waive their rights against experimentation, they retain, at least when they are considered sufficiently capable of exercising it, veto power. (The bar for this capacity is lower than for competence.) The surrogate's consent is usually treated as waiving the subject's rights only so long as the subject also "assents".

The reliance on surrogate consent is a feature of both clinical practice and research. But there is one very striking difference. In clinical practice, apart from exceptional cases such as kidney donations, it is never justifiable to impose net risks on a patient for the benefit of others. But research that imposes risks without an expectation of greater benefit for the subject is conducted not only on subjects capable of consenting to it, but also on incompetent subjects when their surrogate consents. Patients who were previously competent may have expressed a wish when they were competent to make such a sacrifice after they lose competence. Involving them in such a study seems unobjectionable since it accords with their earlier competent wishes, so long as they do not now dissent. But this kind of justification cannot be extended to young children who have never been competent. Permitting children to be exposed to minor research risks for the benefit of other children is relatively uncontroversial in practice. But at the level of justification, it remains puzzling how parental consent could not only waive parental rights against researchers experimenting on their child, but also the child's own right against being exposed to risks for the benefit of others (Ramsey 1976; Wendler 2010).

\subsection{Voluntary consent}

Even when a subject is competent to consent to a research intervention, their consent will be invalid if it is involuntary. Mrs. M, a survivor of experiments that took place at Auschwitz, reports that she agreed to be experimented on by Dr. Mengele only because "As soon as I stood up I was whipped with a leather whip which broke my flesh, then I was told the whipping was a sample of what I would receive if I did not follow instructions and orders" (Claims Conference 2007). Obviously Mrs. M's consent to Mengele's injecting her with drugs and other chemicals did not make it the case she was not wronged by it.

Beyond this uncontroversial sort of case, however, much is in dispute. Some ethicists see involuntariness exemplified in the cases below ${ }^{4}$; others do not. 


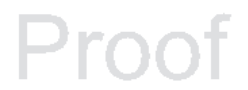

Consent in clinical research

(1) very poor or homeless subjects that consent to research in order to receive a substantial payment;

(2) subjects that are uninsured or under-insured and that consent to research in order to receive health care;

(3) prisoners that consent to research to obtain spending money or better conditions or sentence reductions;

(4) subjects that consent to research because an authority figure in their lives wishes it;

(5) drug addicts that consent to research that involves giving addicts their drug of choice.

One disagreement about voluntariness is just terminological, although if it is not sorted out it can lead to confusion. Everyone agrees that consent may be rendered invalid because of the way the external situation in which the subject chooses is altered, as in Mrs. M's case. Everyone also agrees that consent can be rendered invalid because of the way the subject's ability to recognize or respond to his or her external situation is impaired, as in cases involving the use of deception or temptation. Some authors (Beauchamp 2010; Nelson et al. 2011) prefer a broad conception of "voluntariness" that covers both types of invalidating conditions, whereas others (Appelbaum et al. 2009; Wertheimer 2012) reserve the label for conditions that invalidate because of the way they alter the subject's external situation (see also Gutmann, this volume, Chapter 19). In what follows I will adopt the narrower use of "voluntariness" that confines it to the external situation and discuss the other type of invalidating condition under the label "impairment". Since ethicists that regard the drug addict example (5) above as displaying involuntariness presumably have impairment in mind, we can dismiss it at the outset.

\subsection{The autonomous authorization approach to voluntariness}

With this terminological dispute out of the way, we can focus on the substantive disagreement, which concerns the facts about a subject's external situation that could render their consent involuntary. According to the standard approach in research ethics, the autonomous authorization (AA) approach, a subject's consent is involuntary when it is "substantially controlled" by changes someone else has made to their external situation (Faden et al. 1986; Beauchamp 2010; Nelson et al. 2011). Put another way, whether consent is involuntary depends on the degree of pressure to consent that is brought to bear on their decision by the exercise of someone else's control over their situation. I'll describe the AA approach to involuntariness first, and then introduce a recent competing view. These two views deliver different verdicts about the consent in cases (1)-(4) above.

In Mrs. M's case, the pressure to consent derived from the removal of an option she would have preferred, namely, the option of not participating in research and avoiding a whipping. Following the Belmont Report, advocates of the AA approach tend to label pressure that results from removing more attractive options, as in Mrs. M's case, as potentially "coercive" and pressure that results just from creating more attractive options, like the option of participating in research and making money, receiving access to health care, or other benefits, as potentially "undue inducement". (However, some ethicists (Macklin 1989) and many IRB members (Largent et al. 2012) call pressure from both sources "coercive".) But the AA approach to voluntariness is, ultimately, indifferent to whether the pressure to consent derives from the removal of a more preferable option or just from the creation of a preferable option. At the end of the day, voluntariness is a matter of whether the pressure, no matter its source, is excessive.

When is pressure excessive? According to some adherents of the AA approach, including many IRB members (Largent et al. 2012), an offer of payment or other benefits exerts excessive 
pressure whenever it induces a subject to participate in research. This is a puzzling view, since normally the fact that someone accepts risks to make money is not taken to impugn the voluntariness of their acceptance. What lies behind it, presumably, is Hans Jonas's influential idea that participation in clinical research ought to be intrinsically motivated. Jonas (1969) regards subjects as having been "conscripted" into a study unless they consent to it for the same reason the researcher conducts it, namely, because they identify with the goals of the research. Jonas's view has had a major influence on the way IRBs think about payment for research participation. But it is far from clear why we should treat extrinsically motivated consent to research as involuntary when we treat extrinsically motivated consent to other risky jobs as voluntary.

A less demanding version of the AA approach claims that an incentive is excessive when it leaves the subject with no reasonable alternative but to consent. Notice however that a subject might have no reasonable alternative to participation, not because their status quo is so bad, but only because the benefits of participation are so good. Consider a subject who is financially secure and consents to a risky study in order to receive a payment that vastly exceeds the amount necessary to compensate for the risks. Recall that we are assuming that the subject's consent in these cases is clear-headed. That is, the incentive is not distorting the subject's ability to recognize or respond to their situation, e.g., by blinding the subject to the risks or tempting the subject to accept them against their better judgment. So long as that assumption is kept firmly in mind, it is hard to believe that offering someone who is financially secure substantially more than their reservation price would invalidate their consent.

The AA approach can avoid this problem by treating pressure as excessive only when the subject's alternatives are unacceptable. Financially secure subjects who consent to a risky study offering an extremely generous payment may have no reasonable alternative but to consent, but their status quo is acceptable. A homeless person in need of money or an uninsured person in need of health care, on the other hand, may consent to a study offering payment or health care only because their alternatives are unacceptable. Their consent would still be involuntary, according to this version of the AA approach.

Is this plausible, keeping in mind the assumption that the incentive is not distorting their practical reasoning? A desperate subject may feel forced to consent to a study offering payment or health care by the lack of acceptable alternatives. But a cancer patient also accepts the risks of chemotherapy only because the alternative, certain death, is unacceptable. Surely the cancer patient's consent to chemotherapy is not rendered involuntary by the absence of acceptable alternatives? Granted, there is a difference between the risks of chemotherapy and the risks of research: whereas the benefits of chemotherapy are not detachable from its risks, a researcher could offer money or health care without requiring research participation. But if the researcher does not owe the subject the money or health care without any strings attached, why would conditioning these benefits on research participation render the subject's consent invalid, any more than conditioning the provision of chemotherapy on receipt of payment would render the patient's consent invalid?

\subsection{The moralized approach to voluntariness}

According to the competing approach to voluntariness, what is invalidating about the circumstances of Mrs. M's consent is not that she had no acceptable alternative to consenting, but that the reason she had no acceptable alternative is because the researcher had wrongfully removed an option that she would have preferred, namely, the option of refusing participation and not suffering a whipping. Mrs. $\mathrm{M}$ has a right against being whipped that is not conditional on her 


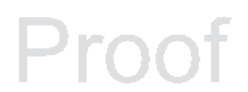

Consent in clinical research

choice about participating in research. Such an account of voluntariness is moralized (Wertheimer 2012). Consent to research is involuntary, according to this kind of approach, when and only when it is caused by the wrongful removal of or refusal to create an option that the subject would have preferred. Creating an option for a subject can never render their consent involuntary, since adding an option cannot wrong the subject, although their consent to that new option will be involuntary if it is a product of the wrongful removal or refusal to create an option they would have preferred.

Mrs. M illustrates how consent can be rendered involuntary by wrongfully removing an option from the subject. Consent is also rendered involuntary when it is prompted by a wrongful failure to create or sustain an option the consenter would have preferred. It is wrong for a physician to abandon a patient. Suppose that a physician decides to motivate her patient to enroll in a study she is conducting by threatening to stop treating him if he refuses. If the patient consents to the study only because of the physician's threat to discontinue an option that he is owed, namely, the option of continuing the physician-patient relationship without participating in the study, then the patient's consent to the study is involuntary.

Whereas the AA approach implies that consent to research that responds to payment is involuntary when the payment is too high or when the subject's need for money is too severe, the moralized approach never implies that an offer of money renders consent involuntary. The payment may help to make it the case that the subject has no reasonable alternative or no acceptable alternative but to consent, but this does not make the consent involuntary. However, the subject's consent to a study that offers payment would be involuntary if it was caused by a wrongful denial of a preferable option. Suppose that a researcher promises a subject that they will receive $\$ 100$ for each stage of a four-stage study that they complete. The subject completes the first two stages, and then decides to withdraw. If, in order to incentivize the subject to complete all four stages, the researcher goes back on the promise and decides that the subject will be given $\$ 400$ only if he completes the two remaining stages and nothing if he drops out now, then the subject's consent to the remaining stages of the study would count as involuntary. It is not the attractiveness of the $\$ 400$ for completing the remaining stages that makes it involuntary, however. Rather, it is the fact that the subject is consenting to the remaining stages only because the subject was wrongfully denied his preferred option, namely, stopping after two stages and receiving $\$ 200$ as promised. ${ }^{5}$

When it comes to the incentive of health care, again, the moralized view will deny that it is ever the offer of health care in itself that renders consent involuntary, since the offer just creates an option and the creation of an option cannot wrong the subject. However, the moralized view does imply that if the subject's consent to research is a product of the researcher's wrongful failure to offer a health care option that the subject would have preferred, then it will be involuntary. A public hospital may owe treatment to patients without strings attached. If it makes participation in a randomized controlled trial a condition on delivery of treatment, the subjects' consent would be involuntary if it is prompted by the hospital's wrongful refusal to offer treatment without research participation. But institutions such as the NIH or private hospitals that do not owe potential subjects treatment outside the context of research can make participation in a trial a condition on delivery of treatment without rendering subjects' consent to the research involuntary (Fried 2016: 43-7). And even public hospitals do not owe patients access to experimental therapies. Suppose that experimental therapy is offered to a patient but only on the condition that they enroll in the clinical trial that is evaluating it. If the therapy offers the subject their last best hope for survival, there would be tremendous pressure on the subject to consent. The subject may much prefer the option of receiving the therapy outside the trial, especially if the trial is placebo-controlled. But insofar as the subject is not owed that 
Collin O’Neil

therapy outside the context of a trial, the subject's consent to the study is not based on the wrongful denial of an option they would have preferred (cf. Schuklenk \& Lowry 2009).

It should now be clear how the moralized approach would handle the case of prison research. If the institution incentivized prisoners to consent to research by threatening to otherwise extend their sentence or remove privileges they had earned, then the prisoner's consent would be involuntary, so long as it was caused by the denial of these options. But if instead the institution offered a reduction in the prisoner's sentence or privileges they were not already entitled to, then the prisoner's consent to the study would be voluntary, since it is not prompted by a removal or denial of an option the prisoner is entitled to.

But suppose the details of the prison research are as follows. The researcher comes in from the outside and seeks volunteers. The researcher has no problem enrolling subjects, but it turns out this is because prison staff (acting on their own and not at the direction of the research team) are threatening to make life worse for prisoners who refuse to sign up. Is their consent still voluntary? A similar concern might lie behind the case of the subject who consents to research under the influence of an authority figure, such as a husband or a tribal leader. This raises an issue about the significance of third-party coercion. On the moralized approach as I've described it, consent to research is involuntary only when it is the product of the researcher's wronging of the subject (Miller \& Wertheimer 2010). (Note that researchers may sometimes have duties to relieve third-party pressure on subjects, and if the subject consents to the study only because the researcher fails to discharge such a duty, the consent would be involuntary.) ${ }^{6}$ The AA approach would not differentiate these cases, since the degree of pressure applied is the same. But there are other versions of a moralized approach that do claim that at least illegitimate third-party coercion can render a subject's consent to the researcher's intervention involuntary even in the absence of any wronging by the researcher (Millum 2014).

\subsection{Informed (unimpaired) consent}

At the beginning of the previous section I distinguished between consent that is invalid because it is involuntary and consent that is invalid because it is impaired. Certain ways of controlling the external situation in which the subject chooses can render consent involuntary. Impaired consent occurs when the subject's ability to recognize and respond to their external situation is distorted. There are a variety of ways in which a researcher could, intentionally or negligently, interfere with a subject's practical reasoning about whether to participate in a study. Although attaching rewards to participation, like payment or health care, cannot as such render consent involuntary, it may interfere with the exercise of subjects' cognitive capacities by blinding them to the risks of the study or their volitional capacities by tempting them into consenting against their better judgment (Wertheimer 2011: 150). ${ }^{7}$ Similarly, the fact that an addict's drug of choice is administered in the study, though not intended as an inducement, could interfere with the exercise of the addict's volitional capacities. (See Walker (2008) for a strategy to obtain consent from addicts that circumvents this problem.) Even making use of the fact that subjects have a hard time saying no to doctors - the so-called "white-coat effect" - could impair the subject's practical reasoning by injecting an irrelevant consideration into their deliberations (Mandava \& Millum 2013).

Attention to the varieties of impairments and the question of when they invalidate consent is an important research program in research ethics (Mandava \& Millum 2013; see Faden et al. (1986) for an early treatment). In this section, however, I will focus on the question that has received the bulk of the attention in the literature on consent, namely, the question of when consent is adequately informed (See also Eyal, this volume, Chapter 24). The autonomous 
authorization (AA) approach embodies the standard answer to this question. But as in the case of voluntariness, the AA approach has come under criticism and competing approaches have been proposed. I will describe these approaches, and draw out their implications concerning the question of whether so-called "therapeutic misconceptions" and other misconceptions invalidate consent.

There is broad agreement that clinical researchers are under duties to disclose extensive information about the study to potential subjects, e.g., about the purpose of the study, what will be done to the subjects, the risks and benefits of the interventions, and appropriate alternatives to participation. In many other contexts in which consent is sought, such as commerce, jobs, sex, and gambling, there is at most a duty not to deceive, or when there are duties to disclose information, they are very modest (Miller \& Wertheimer 2010). In clinical care, however, we traditionally regard clinicians as under a duty to make an extensive disclosure to patients, since it is so hard for patients to learn the relevant facts on their own and relatively easy for the clinicians, who should already know these facts, to disclose them. The same asymmetry applies to researchers and subjects. Indeed, since the primary aim of clinical research is not to benefit the subject but to advance knowledge, researchers are usually regarded as having even more extensive duties of disclosure than clinicians. Since clinicians can be presumed to be acting solely in the patients' best interests, patients arguably have less need for information from their clinicians than potential subjects do from researchers (Chwang 2010).

There is a question about the exact content of the extensive duty of disclosure, e.g., whether it is governed by the professional practice standard, reasonable person standard, or a standard more tailored to each individual subject (Brock 2008). I will assume, for simplicity, that the content of the duty of disclosure is to disclose to the subject all of the facts about the study interventions that the researcher knows or ought to know would be material to the subject's decision. These facts should also be disclosed in a manner that makes them accessible to the subject. Describing the injection in Jewish Chronic Disease Hospital as containing "live neoplastic cells" would not satisfy the duty of disclosure. But the duty of disclosure is only a duty to try to inform the subject, i.e., to give the subject a fair opportunity to become adequately informed. It is not a duty to succeed. Thus it is possible for a researcher to fully comply with their duty of disclosure while the subject remains ignorant of or mistaken about material facts. This raises the question of whether consent based on ignorance or mistake could be valid, so long as it is not attributable to a failure to fully discharge the duty of disclosure.

\subsection{The autonomous authorization approach to informed consent}

Although there is broad agreement about the duty of disclosure, important disagreements emerge when we consider different views about the relation between compliance with the duty of disclosure and the conditions under which ignorance or mistaken beliefs undermine the validity of consent. According to the standard view of informed consent, the autonomous authorization (AA) view, consent is valid only when all of the information about an intervention that is material to the subject's decision to consent is actually understood by the subject (Faden et al. 1986; Beauchamp 2010). ${ }^{8}$ If it were the case that disclosures invariably brought about the corresponding understanding in subjects, then the question of whether this understanding is truly necessary for valid consent would be of merely academic interest. But it turns out that, even after scrupulous disclosures, subjects frequently misunderstand material facts about study interventions and procedures.

One particularly recalcitrant type of misunderstanding is known as the "therapeutic misconception" (Appelbaum \& Lidz 2008). The defining goal of clinical research is to generate 
clinically useful information, not to benefit the subjects of that research. In order to achieve this goal, clinical research frequently involves departures from the individualized care one would expect in a clinical setting. Even when this different purpose and the differences between the design of the study and clinical care are disclosed, subjects frequently continue to expect individualized care in the research setting. For example, they may continue to believe that their treatment will not be selected by a random process, that they would never be given a placebo, that any burdensome or risky intervention must be intended for their direct benefit, and so on. These departures from clinical care will often be material to the subject's decision to consent, at least when the alternative to receiving the therapy in the context of the study is receiving it in clinical practice. Yet these misunderstandings are highly resistant to disclosure.

The AA approach takes the fact that subjects are under a therapeutic misconception as having a straightforward implication for the validity of their consent. Insofar as the subjects are ignorant of or misinformed about material aspects of the study, their consent is invalid, no matter how careful the disclosure was. Given the data about the prevalence of therapeutic misconceptions (Mandava et al. 2012), this in turn implies the rather alarming conclusion that researchers have for years been engaged in the wholesale violation of their subjects' rights. Possibly this conclusion is so implausible as to be reason enough to reject the theory that implies it. But even if not, there is still a powerful reason to rethink the AA theory. Notice that if researchers enhance the disclosures in light of this data, as they should given that the goal of these disclosures is to bring about understanding, there are no measures they could take that would guarantee that subjects are adequately informed. Surely at some point the responsibility for the mistake must belong to the subject, not the researcher. Yet the AA approach does not make any room for this moral possibility.

\subsection{The minimal understanding approach}

Three alternative approaches to the question of when ignorance or mistake on the part of the subject invalidates their consent have recently emerged. Each approach accepts that researchers have an extensive duty of disclosure and that at least a "minimal" understanding of a research intervention is necessary to validly consent to it. Unlike the AA approach, none of these alternatives imply that ignorance or mistake about any material fact undermines the validity of the subject's consent. But they differ as to the conditions under which ignorance or mistake does render a subject's consent invalid.

One kind of approach takes the minimal understanding of an intervention as not only necessary for validity, but also sufficient. A failure to discharge the full duty of disclosure would still wrong the subject, but it would not have the effect of rendering the subject's consent invalid unless as a consequence the subject lacked a minimal understanding of the intervention. What counts as minimal understanding? On the most fully developed version of this approach (Walker 2012; see Sreenivasan 2003 for an earlier version), consenting to a researcher's doing $\mathrm{X}$ requires at a minimum that the subject understand all the descriptions of $\mathrm{X}$ under which doing $\mathrm{X}$ would, without consent, infringe a right of the subject's. The fact that $\mathrm{X}$ would involve bodily contact or invasion is a morally relevant description. So is the fact that it imposes a risk of harm, although only if the level of risk is such that the subject would, independently of the bodily contact, have a right against being exposed to it.

This "minimal understanding" approach is very liberal about ignorance and mistakes concerning research. It seems likely that, on this account, many of the common misunderstandings subjects have about research would not compromise the validity of their consent. But it is in danger of being too liberal about misunderstandings. Suppose that a researcher is seeking a 


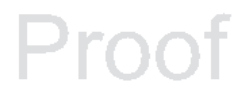

Consent in clinical research

subject's consent to a placebo-controlled randomized trial, which gives her only a $50 \%$ chance of receiving the treatment she needs and that also involves painful non-therapeutic biopsies. In fact, the treatment she needs is readily available outside the trial and if she knew this she would never consent to the trial. The researcher purposefully withholds this crucial information from her. Surely the subject is wronged by the biopsies. But on the minimalist approach, she appears to possess the minimal understanding needed to validly consent to the biopsies. The fact that the treatment she needs is available outside the trial is not a description of the biopsy at all, much less a description of it under which it would wrong her without her consent.

\subsection{The partially moralized approach}

The next two approaches do not regard a minimal understanding of the intervention as sufficient for valid consent (Bromwich \& Millum 2013; Miller \& Wertheimer 2010). Unlike the AA approach, however, these theories do not imply that ignorance of any material fact about the intervention invalidates consent. Ignorance of a material fact renders the subject's consent invalid only if the ignorance is attributable to the researcher's failure to discharge the duty of disclosure. Regarding the case above, both views would regard the subject's ignorance of the availability of treatment outside the trial as invalidating, because this ignorance is due to the researcher's withholding that information in violation of their duty of disclosure. But if the researcher had disclosed this information in a manner that satisfied this duty and the subject still failed to grasp it, the subject's consent would not necessarily be invalidated.

Moralized theories of validity can be compellingly motivated by the following pair of cases (Bromwich \& Millum 2013: 7-8). In the first case, a researcher is aware that the drug she is testing has side-effects $\mathrm{A}$ and $\mathrm{B}$ and discloses both side-effects to the subjects. It is later discovered that the drug also has side-effect $\mathrm{C}$, although no one in the expert community, including the researcher, knew it at the time. Although the subjects are consenting to the drug in ignorance of the material fact that it has side-effect $\mathrm{C}$, their consent seems valid. In the next case, the same researcher, after learning that the drug has side-effect $\mathrm{C}$, initiates another study. She discloses side-effects $\mathrm{A}$ and $\mathrm{B}$ to the subjects, but withholds what she now knows about side-effect $\mathrm{C}$ from them. These new subjects are consenting to the drug in ignorance of the material information about C, just like the previous subjects, but their consent seems clearly invalid. Yet both groups of subjects have the same understanding of the same intervention in both cases.

A plausible explanation for the difference in validity is that the subjects' ignorance of $\mathrm{C}$ in the first case is not attributable to the researcher's failure to discharge their duty of disclosure, whereas the subjects' ignorance in the second case is. On Bromwich's and Millum's view, however, it turns out that only a subclass of the violations of the duty of disclosure can invalidate consent. Returning to the first case, suppose that side-effect $\mathrm{C}$ was well known in the expert community and that the researcher should have known about it, but failed to keep up with the literature. When she fails to disclose $\mathrm{C}$ to the subjects, she is in violation of the duty of disclosure, since this is not merely a duty to disclose what one does know, but what one should know. But they claim that when the failure to discharge the duty is due to ignorance, no matter how negligent, it cannot invalidate consent because it cannot count as an exercise of illegitimate control over the subject's practical deliberations (Bromwich \& Millum 2013: 13).

This account, like the minimal understanding account just discussed, is in danger of being too liberal. Consider a Phase I oncology trial, in which the researcher sincerely tells a cancer patient that the drug has a small but real prospect of arresting the progress of his cancer. In fact, however, patients are to be given only a subtherapeutic dose of the drug in this trial, something the researcher does not know but certainly should have known. In this case, the researcher is 
not merely failing to disclose something important to the patient, but is misinforming the patient about something that would have changed the patient's mind about consenting. Yet, on this approach, this failure to discharge the duty of disclosure would still not invalidate the patient's consent to the trial, because the researcher does not know that she is misinforming the patient, and is therefore not exercising illegitimate control over the subject's decision-making.

\subsection{The fully moralized approach}

If the consent in the oncology case seems invalid, this is a reason to prefer a fully moralized account of validity, like that on offer in Miller \& Wertheimer (2010) and Owens (2007). On such an account, any failure to discharge the duties of disclosure that results in ignorance of a material fact about the intervention would invalidate consent. This account would imply that the consent in the oncology case is invalid. Although the researcher did not know she was failing to discharge her duties of disclosure, she was. And since the subject's mistaken belief was due to this failure, the subject's consent, which was based on that mistaken belief, is invalid. The fully moralized account seems to be able to capture all the cases where, intuitively, consent is invalid, yet without implying, as the AA approach does, that each and every material misunderstanding invalidates consent. ${ }^{9}$

\section{Notes}

1 I use the term "subject" rather than "participant" because the topic of consent is the topic of what is needed to turn a mere subject into a participant.

2 Studies that use deception for certain methodological reasons can also be consistent with respect for subjects' hypothetical consent (O’Neil 2013).

3 The claim that subjects cannot release researchers from certain duties directed towards them is stronger than the uncontroversial claim that consenting to $\mathrm{X}$ does not always make $\mathrm{X}$ permissible, all things considered. E.g., subjects' consent to involvement in a study lacking social value does not release publicly funded researchers from their duty to the taxpayers not to waste resources.

4 These examples are drawn from Appelbaum et al. (2009). These authors are skeptical that (1)-(5) display involuntariness, but see their article for citations of the literature that views (1)-(5) as examples of involuntariness.

5 Suppose that, rather than offering $\$ 400$ for finishing the study, the research ups the offer to $\$ 10,000$, but still denies the subject the promised option of receiving $\$ 200$ after completing two stages. If the subject would prefer to continue the study and receive $\$ 10,000$ rather than stop now and receive the $\$ 200$ he is owed, then the consent is not involuntary. Although the subject is wrongfully denied the option of receiving $\$ 200$ now, the subject's consent to continue on with the study is not caused by the denial of that option. I.e., the subject would have consented to continue the study even if the option owed the subject had been available.

6 I owe this observation to Yulia Gamper.

7 Interestingly, there is evidence that the higher the payment, the more attentive subjects become to the risks of the study (Cryder et al. 2010).

8 They do recognize some exceptions, such as "open withholding" (Faden et al. 1986: 312-3).

9 I am grateful to Marcello DiBello, Andreas Muller, and Peter Schaber for helpful written comments, and to the participants in my Research Ethics seminar at the Center for Bioethics, NYU, Summer 2017, for valuable discussion.

\section{References}

Appelbaum, P.S. and C.W. Lidz (2008) "The Therapeutic Misconception,” in E. Emanuel, C. Grady, R. Crouch, R. Lie, F. Miller and D. Wendler (ed.), The Oxford Textbook of Clinical Research Ethics. New York: Oxford University Press, pp. 633-44.

Appelbaum, P.S., C.W. Lidz and R. Klitzman (2009) "Voluntariness of Consent to Research: A Conceptual Model," Hastings Center Report 39(1): 30-9. 


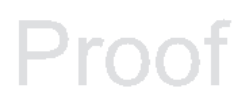

Consent in clinical research

Arras, J.D. (2008) “The Jewish Chronic Disease Hospital Case,” in E. Emanuel, C. Grady, R. Crouch, R. Lie, F. Miller and D. Wendler (ed.), The Oxford Textbook of Clinical Research Ethics. New York: Oxford University Press, pp. 73-9.

Beauchamp, T.L. (2010) "Autonomy and Consent," in F. Miller and A. Wertheimer (ed.), The Ethics of Consent: Theory and Practice. Oxford, New York: Oxford University Press, pp. 55-78.

Brandt, A.M. (1978) "Racism and Research: The Case of the Tuskegee Syphilis Study," The Hastings Center Report 8(6): 21-9.

Brock, D. (2008) "Philosophical Justifications of Informed Consent in Research," in E. Emanuel, C. Grady, R. Crouch, R. Lie, F. Miller and D. Wendler (ed.), The Oxford Textbook of Clinical Research Ethics. New York: Oxford University Press, pp. 606-12.

Bromwich, D. and J. Millum (2013) "Disclosure and Consent to Medical Research Participation," Journal of Moral Philosophy 10(4): 195-219.

Chwang, E. (2010) “A Puzzle about Consent in Research and Practice," Journal of Applied Philosophy 27(3): 258-72.

Claims Conference (2007) "Personal Statements from Victims of Nazi Medical Experiments," Conference on Jewish Material Claims Against Germany, Inc., Last Modified January 1, 2007. Accessed October 16, 2016. www.claimscon.org/about/history/closed-programs/medical-experiments/personal-statements-from-victims/

Cryder, C., A.J. London, K.G. Volpp and G. Loewenstein (2010) "Informative Inducement: Study Payment as a Signal of Risk," Social Science \& Medicine 70: 455-64.

Faden, R.R., T.L. Beauchamp and N.E. Kass (2014) "Informed Consent, Comparative Effectiveness, and Learning Health Care,” New England Journal of Medicine 370(8): 766-8.

Faden, R.R., T.L. Beauchamp and N.M.P. King (1986) A History and Theory of Informed Consent. Oxford, New York: Oxford University Press.

Fried, C. (2016) “Medical Experimentation: Personal Integrity and Social Policy," in F. Miller and A. Wertheimer (ed.). New York: Oxford University Press.

Grady, C. (2015) "Enduring and Emerging Challenges of Informed Consent," New England Journal of Medicine 372(9): 855-62.

Jonas, H. (1969) "Philosophical Reflections on Experimenting with Human Subjects," Daedalus 98 : 219-47.

Karlawish, J.H.T. (2008) “Emergency Research,” in E. Emanuel, C. Grady, R. Crouch, R. Lie, F. Miller and D. Wendler (ed.), The Oxford Textbook of Clinical Research Ethics. New York: Oxford University Press, pp. 280-9.

Largent, E.A., C. Grady, F.G. Miller and A. Wertheimer (2012) "Money, Coercion, and Undue Inducement: Attitudes about Payments to Research Participants," IRB 34(1): 1-8.

Macklin, R. (1989) “The Paradoxical Case of Payment as Benefit to Research Subjects," IRB 11: 1-3.

Mandava, A. and J. Millum (2013) "Manipulation in the Enrollment of Research Participants," Hastings Center Report 43(2): 38-47.

Mandava, A., C. Pace, B. Campbell, E. Emanuel and C. Grady (2012) “The Quality of Informed Consent: Mapping the Landscape. A Review of Empirical Data from Developing and Developed Countries, Journal of Medical Ethics 38(6): 356-65.

Miller, F.G. (2010) “Consent to Clinical Research," in F. Miller and A. Wertheimer (ed.), The Ethics of Consent: Theory and Practice. Oxford, New York: Oxford University Press, pp. 375-404.

Miller, F.G. and A. Wertheimer (2010) "Preface to a Theory of Consent Transactions: Beyond Valid Consent," in F. Miller and A. Wertheimer (ed.), The Ethics of Consent: Theory and Practice. Oxford, New York: Oxford University Press, pp. 79-106.

Millum, J. (2014) "Consent Under Pressure: The Puzzle of Third Party Coercion," Ethical Theory and Moral Practice 17(1): 113-27.

Nelson, R.M., T. Beauchamp, V.A. Miller, W. Reynolds, R.F. Ittenbach and M.F. Luce (2011) “The Concept of Voluntary Consent," American Journal of Bioethics 11(8): 6-16.

O’Neil, C.C. (2013) "Methodological and Inducement Manipulation," American Journal of Bioethics 13(11): $55-7$.

O’Neil, C.C. (2014) “Consent and Rights in Comparative Effectiveness Trials," AMA Journal of Ethics 16(4): 289-94.

Owens, D. (2007) “Duress, Deception, and the Validity of a Promise," Mind 116(462): 293-315.

Owens, D. (2012) Shaping the Normative Landscape. Oxford: Oxford University Press.

Ramsey, P. (1976) “The Enforcement of Morals: Nontherapeutic Research on Children," The Hastings Center Report 6: 21-30. 
Richardson, H. (2012) Moral Entanglements: The Ancillary-Care Obligations of Medical Researcher. Oxford: Oxford University Press.

Rosenstein, D.L. and F.G. Miller (2008) "Research Involving Those at Risk for Impaired Decision-Making Capacity," in E. Emanuel, C. Grady, R. Crouch, R. Lie, F. Miller and D. Wendler (ed.), The Oxford Textbook of Clinical Research Ethics. New York: Oxford University Press, pp. 437-45.

Schuklenk, U. and C. Lowry (2009) "Terminal Illness and Access to Phase 1 Experimental Agents, Surgeries and Devices: Reviewing the Ethical Arguments," British Medical Bulletin 89: 7-22.

Sreenivasan, G. (2003) “Does Informed Consent to Research Require Comprehension?” Lancet 362: 2016-8.

Tadros, V. (2016) Wrongs and Crimes. Oxford: Oxford University Press.

Walker, T. (2008) "Giving Addicts Their Drug of Choice: The Problem of Consent," Bioethics 22(6): 314-20.

Walker, T. (2012) "Informed Consent and the Requirement to Ensure Understanding," Journal of Applied Philosophy 29(1): 50-62.

Wendler, D.S. (2010) The Ethics of Pediatric Research. Oxford: Oxford University Press.

Wertheimer, A. (2011) Rethinking the Ethics of Clinical Research. Oxford: Oxford University Press.

Wertheimer, A. (2012) "Voluntary Consent: Why a Value-Neutral Concept Won't Work," Journal of Medicine and Philosophy 37(3): 226-54.

\section{Related topics}

Ch.7 When is consent required?

Ch.8 Valid consent

Ch.12 Consent and autonomy

Ch.19 Voluntary consent

Ch.24 Informed consent

Ch.25 Consent and medical treatment

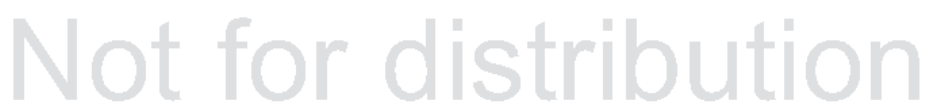

